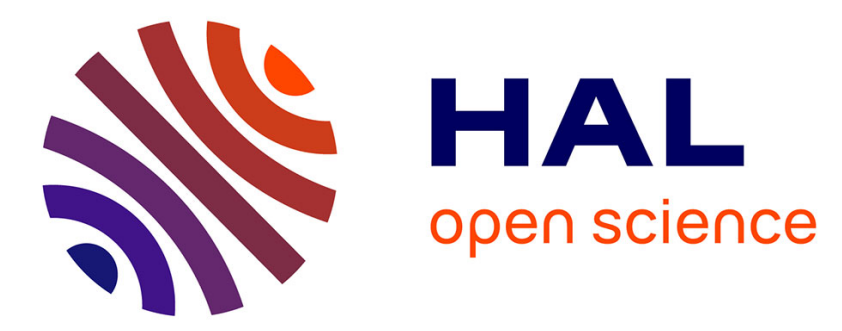

\title{
Development of a hard x-ray wavefront sensor for the EuXFEL
}

Sebastien Berujon, Eric Ziegler, Ruxandra Cojocaru, Thierry Martin

\section{To cite this version:}

Sebastien Berujon, Eric Ziegler, Ruxandra Cojocaru, Thierry Martin. Development of a hard x-ray wavefront sensor for the EuXFEL. SPIE Optics + Optoelectronics 2017, Apr 2017, Prague, Czech Republic. pp.102370K, 10.1117/12.2269452 . hal-01815187

\section{HAL Id: hal-01815187 \\ https://hal.science/hal-01815187}

Submitted on 17 Jul 2018

HAL is a multi-disciplinary open access archive for the deposit and dissemination of scientific research documents, whether they are published or not. The documents may come from teaching and research institutions in France or abroad, or from public or private research centers.
L'archive ouverte pluridisciplinaire HAL, est destinée au dépôt et à la diffusion de documents scientifiques de niveau recherche, publiés ou non, émanant des établissements d'enseignement et de recherche français ou étrangers, des laboratoires publics ou privés. 


\title{
Development of a hard X-ray wavefront sensor for the EuXFEL
}

\author{
Sebastien Berujon*a, Eric Ziegler ${ }^{\text {a }}$, Ruxandra Cojocaru ${ }^{\text {a }}$, Thierry Martin ${ }^{\text {a }}$ \\ ${ }^{a}$ European Synchrotron Radiation Facility, CS 40220, F-38043 Grenoble cedex 9, France
}

\begin{abstract}
We present developments on a hard X-ray wavefront sensing instrument for characterizing and monitoring the beam of the European X-ray Free Electron Laser (EuXFEL). The pulsed nature of the intense X-ray beam delivered by this new class of facility gives rise to strong challenges for the optics and their diagnostic. In the frame of the EUCALL project Work Package 7, we are developing a sensor able to observe the beam in the X-ray energy range [8-40] keV without altering it. The sensor is based on the speckle tracking principle and employs two semi-transparent optics optimized such that their X-ray absorption is reduced. Furthermore, this instrument requires a scattering object with small random features placed in the beam and two cameras to record images of the beam at two different propagation distances. The analysis of the speckle pattern and its distortion from one image to the other allows absolute or differential wavefront recovery from pulse to pulse. Herein, we introduce the stakes and challenges of wavefront sensing at an XFEL source and explain the strategies adopted to fulfil the high requirements set by such a source.
\end{abstract}

Keywords: wavefront, sensor, diagnostic, X-ray Free Electron Laser, metrology, speckle

\section{INTRODUCTION}

Hard X-rays are simultaneously light waves with wavelengths in the Angstrøm range and photons with energies of several kilo electron-volts, such properties making this light very difficult to handle. While the optics requires a precision of the optical figure approaching such a short wavelength, the optical material interacts only weakly with the photons. This is why only few approaches are available for wavefront sensing in the hard X-ray regime, where the deflection of light involves angular magnitudes that are much smaller than one degree. Yet, wavefront sensing becomes meaningful on condition that the sensing accuracy is better than the wavelength.

Usually, the spatial resolution required for an X-ray at-wavelength metrology tool should be of a few micrometers and its wavefront gradient sensitivity on the order of a few tens of nanoradian. Several approaches fulfilling these precision requirements have been developed at synchrotron sources ${ }^{1}$. Nevertheless, most of them rely on the generally true assumption that synchrotrons deliver stable beams, thus making the acquisition of many measurements possible.

Hard X-ray wavefront sensing of a XFEL beam is more intricate than for an X-ray synchrotron beam due to the pulsed nature of the delivered light. Each bunch of photons is generated independently and presents optical features that must be characterized. Several wavefront characterization techniques were tested at XFEL facilities including the grating-based ${ }^{2-4}$ and the coherent diffraction imaging-based methods ${ }^{5-7}$.

One drawback of grating-based methods is the absorption induced by the regular wavefront modulator and the hypothesis stating that the grid would remain perfect and unruffled during the acquisition of a series of measurements over a train of $\mathrm{X}$-ray pulses. A sine qua non condition for such an assumption to be valid is that gratings are made of a stable material such as diamond. The silicon grating, commonly employed with a synchrotron beam, would be too sensitive to the high flux density delivered by the XFEL. Diffraction-based methods requires many exposures to achieve the accuracy and certainty necessary for the optimization of a beamline, which eventually can make these methods slow to converge.

In the frame of the EUCALL project, a consortium of European institutes providing pulsed X-ray light sources, the ESRF is committed to develop a wavefront sensor based on the speckle tracking method capable of sensing the wavefront of each individual hard X-ray pulse arriving at the detector. The instrument is planned to be installed at the FXE beamline ${ }^{8}$ of the EuXFEL for the commissioning campaigns of the instrument. 
The beam provided by the new EuXFEL facility in Hamburg will feature exceptional properties in terms of power, coherence and pulse length. The EuXFEL will operate in a quasi-burst mode, the pulse being organized in trains with a repetition rate of $10 \mathrm{~Hz}$ and a structure made of pulses generated at a frequency up to $4.5 \mathrm{MHz}$.

In the hard X-ray range, each pulse will be loaded with an energy rate up to $10 \mathrm{~kW}$. Hence, huge thermal constrains are placed on the optics. For instance, the optical state could be modified between the first and last pulses of a train due the energy deposited in the optical system. The need for a wavefront sensor capable of analyzing the pulse state dynamically is therefore a high stake for the full exploitation of the FEL beam.

\section{SPECKLE-BASED WAVEFRONT SENSOR PRINCIPLE}

The sensor developed in the frame of the EUCALL project employs the speckle tracking principle introduced a few years ago at synchrotrons for absolute wavefront metrology. The term 'absolute metrology' refers to a metrology that accounts for all the distortions occurring on the optical beamline components, eventually mapping the global state of the wavefront beam with a metric reproducible in time and space.

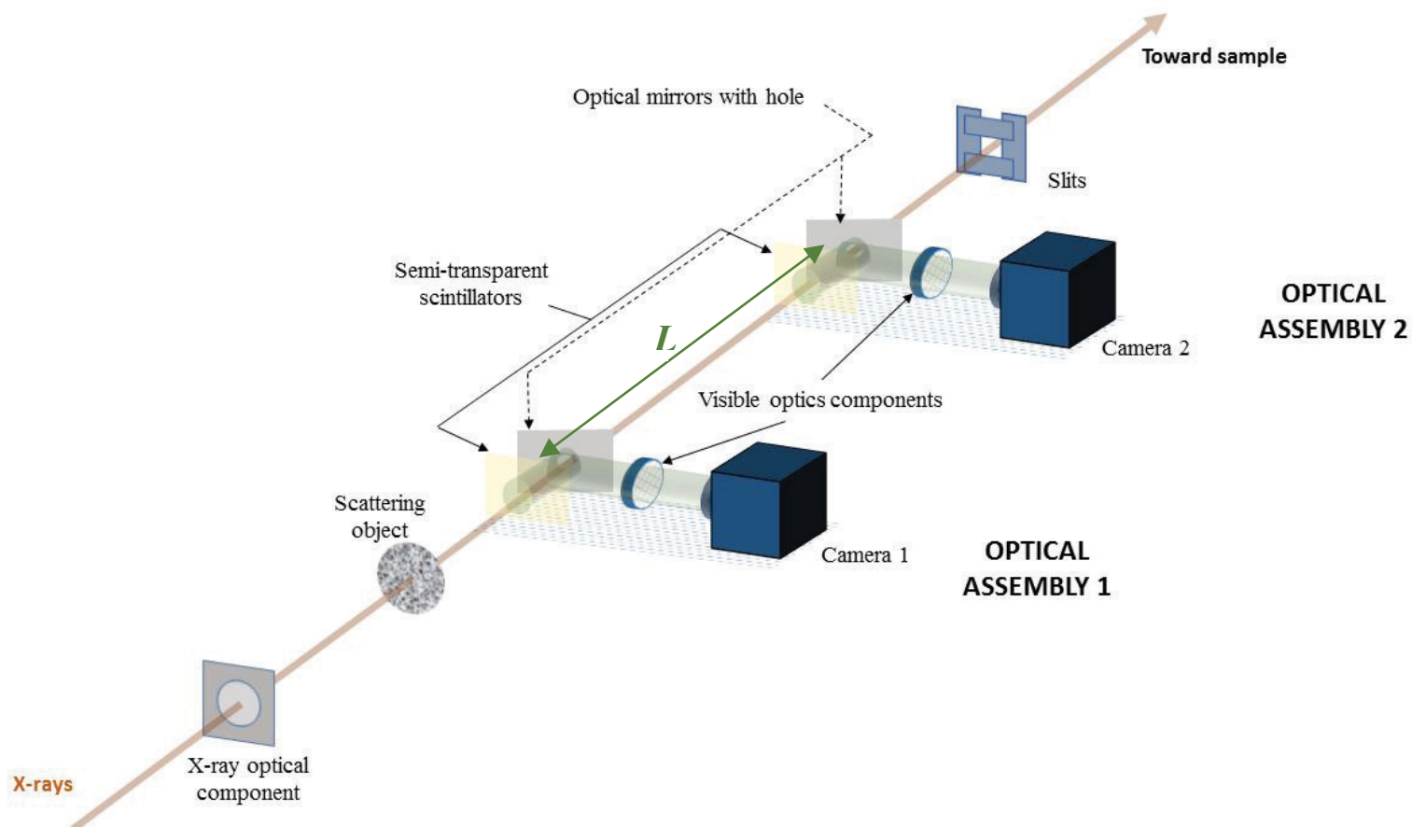

Figure 1. Sketch of the speckle based wavefront sensor. Two imaging systems are looking at the X-ray beam that is modulated with an intensity pattern thanks to a scattering object with small random features. Thin scintillators and low absorbing or holed visible mirrors permit to reduce the absorption of each imaging system.

The sensor principle is sketched in Figure 1. The wavefront is modulated in intensity by a scattering object that creates mutual interference between the scattered photons and the ones which did not interact with the object. This interference creates a random pattern, called speckle that, in the near field regime, has the particularity of having its shape transformation upon propagation to be solely ruled by the wavefront distortion. Next, two semi-transparent imaging detectors record pair of images of this object at two different propagation distances. Then, the remaining part of the beam, i.e not absorbed within the process, propagates further downstream to a sample or a second experiment. 
The X-ray speckle tracking ${ }^{9}$ principle permits to recover the wavefront gradient through the calculation of the local deflection angle of the speckle modulating pattern $\alpha_{P}$. By noting the distance between the two scintillator screens $L$, the local wavefront derivative $\nabla W$ is recovered using the equation:

$$
\alpha_{P}=\nabla W=\frac{\vec{v}}{L}
$$

where $\vec{v}$ is the local displacement of the speckle pattern, within a scalar factor. The principle of the X-ray speckle tracking technique consists of taking a subset of the image collected on the first detector and finding its counterpart in the image recorded on the second detector by using a cross-correlation algorithm to infer $\vec{v}$ and hence $\nabla W$. The resolution of the processing method was shown to approach a few pixels in size and the angular sensitivity to reach a fraction of pixel divided by the distance $L$.

\section{IMPLEMENTATION AND RESULTS}

A prototype is currently being developed at the beamline BM05 of the ESRF and was tested at both the BM05 and ID06 beamlines. The sensor will be later deployed at the FXE beamline of the EuXFEL during the instrument commissioning stage. The data presented here was obtained at the ID06 beamline where, for early development purposes, only the first imaging system was semi-transparent to X-rays. For the tests performed at ID06 the detectors consisted of the combination of a PCO 2000 and a FReLoN camera whilst the setup used at BM05 is presently accommodating two PCO Edge cameras as the one shown in Figure 2. These cameras can achieve a frequency rate of $50 \mathrm{~Hz}$ with $2 \mathrm{k}$ x $2 \mathrm{k} 16$-bit images and low electronic noise. However, given that the EuXFEL X-ray pulse rate is able to reach $4.5 \mathrm{MHz}$, commercially available ultrafast imaging camera are being considered for the sensor deployment there. Such fast cameras are expected to enable the discrimination of each XFEL pulse, providing a better depiction of the thermal and optical variations across the train pulses.

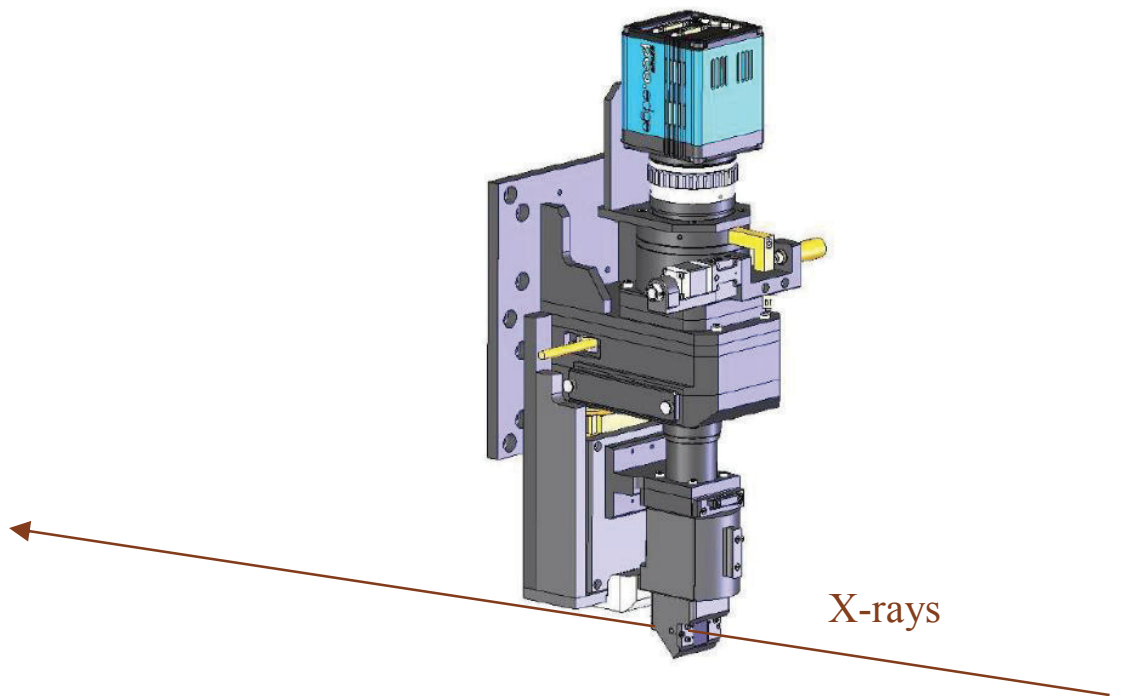

Figure 2. Mechanical assembly of the semi-transparent optics. When the the X-ray beam passes through the scintillator, luminescent light is reflected toward an imaging detector whilst the non-absorbed X-rays continue their propagation toward the second imaging detector.

Figure 2 shows a drawing of the semi-transparent optics used for the first detector. The mechanical assembly was designed and produced by the company Optique Peter. The X-ray beam passes through a thin scintillator whose light produced by luminescence is reflected at ninety degrees with respect to the beam direction by a thin vitreous carbon mirror toward a 
magnifying optics and the CCD camera. A choice of two fast scintillators has been identified: a thin YAG:Ce (70 ns decay time) crystal and an in-house developed LYSO:Ce (40 ns decay time) scintillator able to operate efficiently at high X-ray energy. The thinning of these scintillators is of paramount importance to reduce the amount of X-rays absorbed within the scintillator. It helps prevent any damage that could be caused by the interaction of the scintillator with the intense X-ray beam contained in the X-ray pulse train of the EuXFEL source.

In this implementation, the scattering object was a $120 \mu \mathrm{m}$ thick membrane made of cellulose acetate. At the working energy, the absorption of this object was only of a couple of percent. However, such material cannot sustain the high flux of the EuXFEL over several pulses. For the device implementation with high flux density X-ray sources, solutions are envisaged based either on a diamond modulator or on a liquid jet injector for renewing the modulating material.
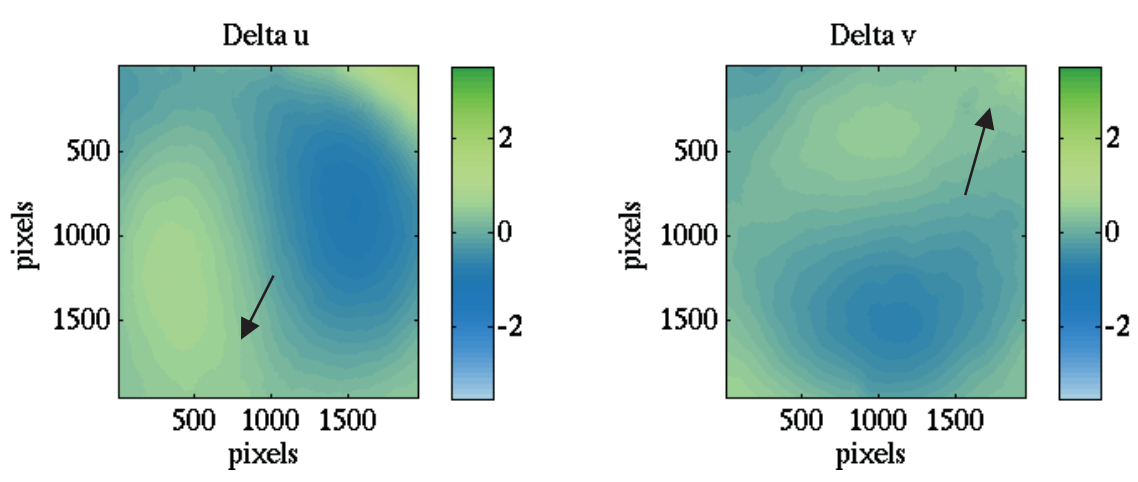

Figure 3. Calculated distortion for the first imaging system: the combined effect of the scintillator mount and of the semitransparent mirror generates a distortion that is not fully radial as pointed out by the arrows.

In the ID06 setup, the first detector was located at a distance of $55 \mathrm{~m}$ from the source and the second one at a distance $\mathrm{L}=$ $450 \mathrm{~mm}$ further downstream from the first detector. As mentioned earlier, the second imaging camera used at the ESRF ID06 beamline was of a more traditional conception where all the photons are absorbed within the imaging system. The detector distortions were characterized and compensated using the method described in Ref. ${ }^{10}$. The calculated distortion for the first detector is shown in Figure 3. One can notice that the recovered distortion is not purely radial and hence does not follow the often-used Brown-Conrady model for optical distortion ${ }^{11}$. This is mainly due to the X-ray transparent mirror that creates optical aberrations upon reflection. In this context, the zonal model approach described in Ref. ${ }^{10}$ is better suited to correct the distortions in the acquired images. The calculated effective pixel sizes were of $0.62 \mu \mathrm{m}$ for the first detector and of $0.74 \mu \mathrm{m}$ for the second one.

The tests conducted at these beamlines aimed at characterizing the ability of the sensor to recover the wavefront curvatures and the source distance in order to infer the optical aberrations of the optics. The photons of the beamline where selected around $\mathrm{E}=17 \mathrm{keV}$ thanks to a double-crystal $\mathrm{Si}(111)$ monochromator.

Figure 4 shows an example of two images recorded on the detector during the same pulse generated using a shutter. The calculated wavefront gradients are shown in (c) and the wavefront reconstruction obtained by 2D integration is shown in (d). One phenomenon observable in the zoomed insets of (b) is that the speckle grains have a much larger shape in the horizontal direction than in the vertical one. This phenomenon, even more pronounced on the image coming from the second detector, arises due to the lower transverse coherence of the high-keta undulator source in the horizontal direction with respect to the vertical one. It is responsible for the blurring of the speckle upon propagation over a distance larger than thı; (leep Fresnel region ${ }^{12}$. 

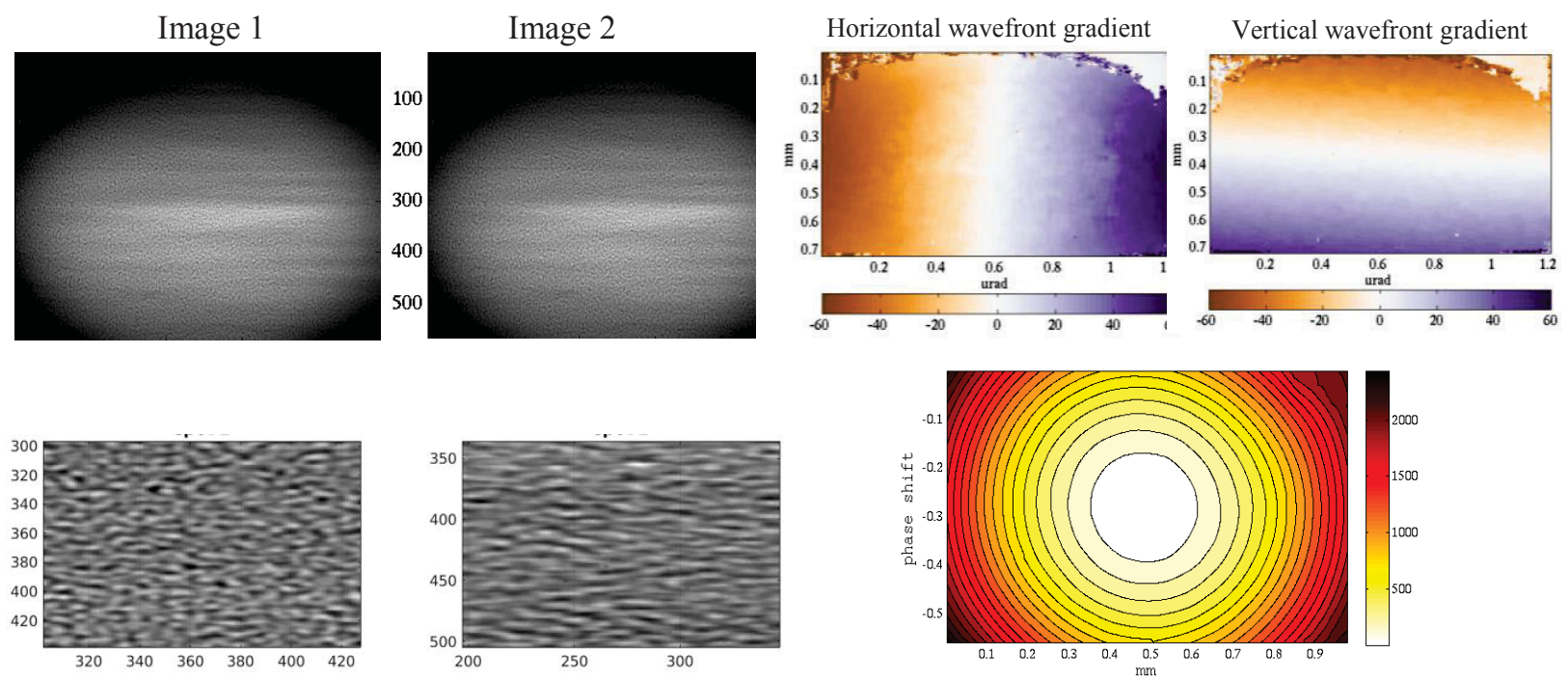

Figure 4. (a) Example of a pair of images collected with the two detectors (case 3 in the next table). (b) Inset of the images showing the light modulation with speckle. (c) Calculated wavefront gradients and (d) wavefront reconstruction.

The X-ray beam state was changed by using different lens combinations offered by a beamline focusing apparatus based on motorized sets of compound refractive lenses, also called transfocator ${ }^{13}$. This transfocator, located at a distance of $38.6 \mathrm{~m}$ from the source, possesses different lens assemblies that can be aligned into the beam either individually or by stack. The focusing power of a lens stack, defined by:

$$
\frac{1}{f}=2 \delta \sum_{i} \frac{N_{i}}{R_{i}}
$$

where $\delta$ is the refraction index of the compound material, $N_{i}$ is the number of lenses and $R_{i}$ their radius of curvature, was then compared to the measured one. The theoretical and measured focusing powers are given in Table 1.

Table 1. Theoretical and recovered focusing power for the different transfocator configurations.

\begin{tabular}{|c|c|c|c|c|}
\hline $\begin{array}{c}\text { Transfocator } \\
\text { Lens Configuration }\end{array}$ & $\begin{array}{c}\text { Transfocator } \\
\text { Lens }\end{array}$ & $\begin{array}{l}\text { Focusing power } 1 / \mathrm{f}= \\
2 \delta \Sigma\left(\mathrm{N}_{\mathrm{i}} / \mathrm{R}\right)\left[10^{-5} \mathrm{~mm}^{-1}\right]\end{array}$ & $\begin{array}{l}\text { Calc. 1/f Horiz. } \\
\qquad\left[10^{-5} \mathrm{~mm}^{-1}\right]\end{array}$ & $\begin{array}{c}\text { Calc. } 1 / \text { f Vert. } \\
{\left[10^{-5} \mathrm{~mm}^{-1}\right]}\end{array}$ \\
\hline 3 & 1 Lens $x 1.5 \mathrm{~mm}$ radius & 0.16 & 0.07 & 0.09 \\
\hline 4 & 1 Lens $x 1.0 \mathrm{~mm}$ radius & 0.24 & 0.14 & 0.13 \\
\hline 5 & 1 Lens $\mathrm{x} 0.5 \mathrm{~mm}$ radius & 0.47 & 0.50 & 0.19 \\
\hline 6 & 1 Lens $\times 0.3 \mathrm{~mm}$ radius & 0.79 & 0.61 & 0.34 \\
\hline 7 & 1 Lens $\mathrm{x} 0.2 \mathrm{~mm}$ radius & 1.18 & 0.91 & 0.52 \\
\hline 8 & 2 Lenses $\times 0.2 \mathrm{~mm}$ radius & 2.36 & 2.42 & 1.06 \\
\hline 9 & 4 Lenses $\times 0.2 \mathrm{~mm}$ radius & 4.72 & 5.2 & 3.05 \\
\hline
\end{tabular}

In Table 1, one can see that the calculated focusing power increases following closely the theoretical predictions both for the vertical and horizontal directions. Some of the discrepancies may be attributed to the lenses aberrations. The offset between the vertical and horizontal directions relates to the source asymmetry. Such an experiment validates the approach for the characterization of pulses with various wavefront curvatures to define the origin of the X-ray source in the FEL undulator. The current prototype accessing the data acquisition capabilities allows one to reach a frequency rate of $50 \mathrm{~Hz}$ whilst computing developments are now aiming at increasing the processing speed to this frequency and thus achieving real time feedback on the pulse wavefront state. 


\section{CONCLUSION}

We presented here efforts deployed at the ESRF for the development and tuning of a wavefront sensor prototype able to operate in the hard X-ray range with ultra-intense pulses. The device will be used as a tool to monitor and optimize the EuXFEL beam in a near future commissioning stage. The sensor will permit to gain unique insight on the optics behavior and allow real time feedback on the X-ray pulse state thanks to GPU optimized processing codes. Such a device will be also instrumental in the monitoring and optimization of future new quasi-CW FEL sources such as LCLS-II ${ }^{14}$. Additionally, the project aims at developing an open-source software package that will be released by the end of 2018 for hard X-ray wavefront sensing based on speckle modulation.

\section{ACKNOWLEDGMENTS}

RC acknowledges support from the European Cluster of Advanced Laser Light Sources (EUCALL) project which has received funding from the European Union's Horizon 2020 research and innovation program. The authors wish to thank Carsten Detlefs for providing access and support at the ID06 beamline, Roberto Homs and Manuel Perez for the technical support and the ESRF for financial and personal support.

\section{REFERENCES}

[1] K. Sawhney, H. Wang, J. Sutter et al., "At-wavelength Metrology of X-ray Optics at Diamond Light Source," Synchrotron Radiation News, 26(5), 17-22 (2013).

[2] S. Rutishauser, L. Samoylova, J. Krzywinski et al., "Exploring the wavefront of hard X-ray free-electron laser radiation," Nat Commun, 3, 947 (2012).

[3] Y. Kayser, S. Rutishauser, T. Katayama et al., "Shot-to-shot diagnostic of the longitudinal photon source position at the SPring-8 Angstrom Compact Free Electron Laser by means of x-ray grating interferometry," Optics Letters, 41(4), 733-736 (2016).

[4] Y. Kayser, S. Rutishauser, T. Katayama et al., "Wavefront metrology measurements at SACLA by means of Xray grating interferometry," Optics Express, 22(8), 9004-9015 (2014).

[5] N. D. Loh, D. Starodub, L. Lomb et al., "Sensing the wavefront of x-ray free-electron lasers using aerosol spheres," Optics Express, 21(10), 12385-12394 (2013).

[6] A. Schropp, R. Hoppe, V. Meier et al., "Full spatial characterization of a nanofocused x-ray free-electron laser beam by ptychographic imaging," Scientific Reports, 3, 1633 (2013).

[7] M. Sikorski, S. Song, A. Schropp et al., "Focus characterization at an X-ray free-electron laser by coherent scattering and speckle analysis," Journal of Synchrotron Radiation, 22(3), 599-605 (2015).

[8] C. Bressler, A. Galler, and W. Gawelda, "Technical Design Report: Scientific Instrument FXE,” XFEL. EU TR2012-008, (2012).

[9] S. Berujon, E. Ziegler, R. Cerbino et al., "Two-Dimensional X-Ray Beam Phase Sensing," Physical Review Letters, 108(15), 158102 (2012).

[10] S. Berujon, E. Ziegler, and P. Cloetens, "X-ray pulse wavefront metrology using speckle tracking," Journal of Synchrotron Radiation, 22(4), 886-894 (2015).

[11] D. C. Brown, "Decentering Distortion of Lenses," Photometric Engineering, 32(3), 444-462 (1966).

[12] R. Cerbino, "Correlations of light in the deep Fresnel region: An extended Van Cittert and Zernike theorem," Physical Review A, 75(5), 053815 (2007).

[13] G. B. M. Vaughan, J. P. Wright, A. Bytchkov et al., "X-ray transfocators: focusing devices based on compound refractive lenses," Journal of Synchrotron Radiation, 18(2), 125-133 (2011).

[14] L.-I. D. S. Group, [LCLS-II conceptual design report] Report LCLSII-1.1-DR-0001-R0, SLAC, (2014). 